\title{
Perfluoroheptanoic acid affects amphibian embryogenesis by inducing the phosphorylation of ERK and JNK
}

\author{
MIRAN KIM $^{1 *}$, MI SEON PARK ${ }^{2 *}$, JUNGEUN SON ${ }^{1}$, INJI PARK ${ }^{1}$, HYUN-KYUNG LEE $^{1}$, \\ CHOWON KIM ${ }^{1}$, BYUNG-HWA MIN ${ }^{2}$, JAEWOONG RYOO ${ }^{1}$, KWANG SHIK CHOI ${ }^{1}$, \\ DONG-SEOK LEE ${ }^{1}$ and HYUN-SHIK LEE ${ }^{1}$
}

\begin{abstract}
${ }^{1}$ ABRC, CMRI, School of Life Sciences, College of Natural Sciences, BK21 Plus KNU Creative BioResearch Group, Kyungpook National University, Daegu 702-701; ${ }^{2}$ Aquaculture Management Division, National Fisheries Research and Development Institute, Busan 619-705, Republic of Korea
\end{abstract}

Received June 12, 2015; Accepted October 1, 2015

DOI: $10.3892 / \mathrm{ijmm} .2015 .2370$

\begin{abstract}
Perfluoroalkyl compounds (PFCs) are globally distributed synthetic compounds that are known to adversely affect humanhealth.Developmental toxicity assessment ofPFCs is important to facilitate the evaluation of their environmental impact. In the present study, we assessed the developmental toxicity and teratogenicity of PFCs with different numbers of carbon atoms on Xenopus embryogenesis. An initial frog embryo teratogenicity assay-Xenopus (FETAX) assay was performed that identified perfluorohexanoic (PFHxA) and perfluoroheptanoic (PFHpA) acids as potential teratogens and developmental toxicants. The mechanism underlying this teratogenicity was also investigated by measuring the expression of tissue-specific biomarkers such as phosphotyrosine-binding protein, xPTB (liver); NKX2.5 (heart); and Cyl18 (intestine). Whole-mount in situ hybridization, reverse transcriptase-polymerase chain reaction (RT-PCR), and histologic analyses detected severe defects in the liver and heart following exposure to PFHxA or PFHpA. In addition, immunoblotting revealed that PFHpA significantly increased the phosphorylation of extracellular signal-regulated kinase (ERK) and c-Jun N-terminal kinase (JNK), while PFHxA slightly increased these, as compared with the control. These results suggest that $\mathrm{PFHxA}$ and $\mathrm{PFHpA}$ are developmental toxicants and teratogens, with $\mathrm{PFHpA}$ producing more severe effects on liver and heart development through the induction of ERK and JNK phosphorylation.
\end{abstract}

Correspondence to: Professor Hyun-Shik Lee, ABRC, CMRI, School of Life Sciences, College of Natural Sciences, BK21 Plus KNU Creative BioResearch Group, Kyungpook National University, Daegu 702-701, Republic of Korea

E-mail: leeh@knu.ac.kr

${ }^{*}$ Contributed equally

Key words: perfluoroalkyl compounds, developmental toxicity, teratogenicity, Xenopus, embryogenesis

\section{Introduction}

Environmental pollution emerged as a significant threat to human health early in the 20th century, as industrial societies became aware of an increased prevalence of a range of chronic illnesses and conditions. Environmental pollution is fueled by large-scale generation of synthetic chemicals that are normally employed in necessary industrial processes $(1,2)$. Among these anthropogenic chemicals, perfluoroalkyl compounds (PFCs) are organofluorines, which have fluorine instead of hydrogen in their carbon chains. Strong bonds form between fluorine and carbon (Fig. 1A). The carbon-fluorine (C-F) bond is considered the strongest polar covalent bond, with a high dissociation energy reaching $117 \mathrm{kcal} / \mathrm{mol}$. The strong polarity and high dissociation energy of C-F bonds make PFCs resistant to a range of biochemical processes such as thermal, chemical, hydrolytic, and biological degradation (2). Furthermore, the hydrophobic fluorinated alkyl chain and the lipophilic functional group make PFCs valuable in many industrial and commercial processes involved in the production of fabrics, electronics, pesticides, emulsifiers, paints and adhesives $(3,4)$. This wide-ranging use of perfluorohexanoic (PFHxA) and perfluoroheptanoic (PFHpA) acids has resulted in their detection as pollutants in water, sediments, air, wildlife and humans, and these compounds are not degraded by any naturally occurring processes in the environment (5).

PFCs are present in atmospheric and aquatic systems on a global level (6). Environmental levels of PFCs were investigated in different world regions, identifying high PFC levels reaching $100 \mathrm{ng} / \mathrm{l}$ (7-9). The concentrations of various PFCs, which exert unpleasant effects in humans, have been determined in human blood samples from different world regions (10). High levels of PFCs in serum and liver samples from both humans and animals have also been detected (11). The levels of PFCs were elevated in individuals with regular occupational exposure to PFCs (4.3 ppb), as compared to the general population (12). Their unique physicochemical properties make PFCs highly persistent, increasing their bioaccumulation and toxicological effects $(5,13)$. In addition, it has been shown that PFCs exert toxic reproductive and developmental effects on human and animal systems $(12,14,15)$. 
Overexposure to these anthropogenic chemicals causes severe fetal abnormalities in amphibians and affects human development $(12,16)$. The toxicity of PFCs is commonly determined by their carbon chain length and by the functional group.

The frog embryo teratogenicity assay-Xenopus (FETAX) utilizing anuran Xenopus embryos is a well-characterized method used to measure the developmental toxicity of chemicals. The FETAX assay is performed on fertilized Xenopus embryos in the middle of the blastula stage, at $96 \mathrm{~h}$ (17). Teratogenic effects are assessed by determining their influences on mortality, malformation, and growth inhibition, effects that clearly indicate developmental toxicity. This assay has been used to investigate teratogens and developmental toxicants that negatively affect human health and the ecosystem $(18,19)$. FETAX is appropriate for the screening of chemicals (including nanomaterials), evaluating biotransformation, and can serve as a model of limb development. It is used to inform risk assessments of the effects of environmental hazards on amphibian and vertebrate populations. In addition to FETAX, reverse transcriptase-polymerase chain reaction (RT-PCR) and whole-mount in situ hybridization are useful in the investigation of the developmental toxicity of these compounds, as well as mechanisms underlying any effects on Xenopus embryos.

The toxicological potentials of PFHxA and PFHpA need to be investigated to determine the adverse effects of PFCs with different lengths. In the present study, we evaluated the developmental toxicity and teratogenicity of PFHxA and PFHpA using different biological assays. The results obtained from the in vivo model system showed that $\mathrm{PFHpA}$ is a more potent developmental toxicant and teratogen than PFHxA. In particular, PFHxA and PFHpA affected mortality and the growth rates of embryos, and caused severe developmental liver and heart defects. These results suggested that although PFHxA and PFHpA are developmental toxicants and teratogens, PFHpA has more severe effects on liver and heart development via the induction of extracellular signal-regulated kinase (ERK) and c-Jun N-terminal kinase (JNK) phosphorylation.

\section{Materials and methods}

Ethics statement. We obtained a specific waiver from the Institutional Review Board of Kyungpook National University for the experimental use of amphibians or reptiles in Korea (no. KNU-2013-222). All the members of our research group attended educational and training courses on the appropriate care and usage of experimental animals. Adult Xenopus laevis were entrained in 12-h light/dark cycles at $18^{\circ} \mathrm{C}$ in containers provided by the Institutional Review Board of Kyungpook National University constructed to specifications for laboratory animal maintenance. There were no unexpected deaths of adult Xenopus during the study.

Chemicals and reagents. PFHxA and PFHpA were purchased from Sigma-Aldrich (St. Louis, MO, USA) and dissolved in dimethyl sulfoxide (DMSO) to produce $1 \mathrm{M}$ stock solutions. The FETAX medium used in the experiment contained $10.7 \mathrm{mM} \mathrm{NaCl}, 1.14 \mathrm{mM} \mathrm{NaHCO}, 0.4 \mathrm{mM} \mathrm{KCl}, 0.1 \mathrm{mM}$ $\mathrm{CaCl}_{2}, 0.35 \mathrm{mM} \mathrm{CaSO}{ }_{4} \cdot 2 \mathrm{H}_{2} \mathrm{O}$, and $0.3 \mathrm{mM} \mathrm{MgSO}_{4}$ and the levels of DMSO did not exceed $0.15 \%$, which was below the permissible level for this assay. PFC stocks were freshly made and diluted in FETAX medium.

In vitro fertilization. Experiments on Xenopus laevis were conducted in accordance with documented standards of the Animal Care and Use Committee, in agreement with international laws and policies [National Institutes of Health (NIH) Guide for the Care and Use of Laboratory Animals, NIH publication no. 85-23, 1985]. Adult Xenopus were purchased from Nasco (Fort Atkinson, WI, USA) and kept in climate-controlled clear plastic aquariums with dechlorinated tap water at $18 \pm 2^{\circ} \mathrm{C}$, with 12 -h light/dark cycles. The housed frogs were fed only three times a week. For the induction of ovulation, human chorionic gonadotropin (1,000 IU) (Sigma-Aldrich) was injected under the skin of a female Xenopus in the evening. The following day, the females were made to lay eggs in 60 -mm plastic dishes. The eggs were immediately fertilized in 0.1X modified Barth's saline (MBS) containing $88 \mathrm{mM} \mathrm{NaCl}, 5 \mathrm{mM}$ Hepes, $2.5 \mathrm{mM} \mathrm{NaHCO}_{3}$, $1 \mathrm{mM} \mathrm{KCl}, 1 \mathrm{mM} \mathrm{MgSO}$, and $0.7 \mathrm{mM} \mathrm{CaCl}_{2}$ (pH 7.8) after washing three times with $0.1 \mathrm{X} \mathrm{MBS}$. The frogs were sacrificed as follows: The anesthetic of choice for male frogs, tricaine methanesulfonate (MS222) was dissolved in dechlorinated water in an induction tank at a dose of $500 \mathrm{mg}$ of MS222/1 of water. Xenopus testes were obtained from the sacrificed males and minced in $1 \mathrm{ml}$ of cold $1 \mathrm{X}$ MBS. Following successful fertilization, the jelly coat was removed from the embryos by gentle swirling in a $2 \%$ L-cysteine solution. The embryos were then transferred to $1 \mathrm{X}$ MBS containing 3\% Ficoll 400 (GE Healthcare, Little Chalfont, UK). Unfertilized eggs and dead embryos were removed and the remaining embryos were maintained at $22 \pm 0.5^{\circ} \mathrm{C}$ until they reached blastula stage 8.5.

FETAX assay. FETAX assays were conducted to assess the developmental toxicity and teratogenic effects of the PFCs based on the American Society of Testing Material (ASTM) guide (ASTM E1439-98). Finely cleaved embryos in the blastula stage 8.5 were selected and used to exclude the effects of spontaneous embryonic developmental problems. Embryos were impartially allocated to $100-\mathrm{mm}$ petri dishes (20-25 embryos/dish) and exposed to different concentrations of PFHxA $(0.1,0.5,1,1.5$ and $2 \mathrm{mM})$ and PFHpA $(0.25,0.5,0.75,1$ and $1.25 \mathrm{mM})$. DMSO $(0.1 \%)$ and FETAX medium alone were used as the controls. Embryos were incubated at $23^{\circ} \mathrm{C}$ until the end of the assay, and the media were changed daily and dead embryos were removed. At the end of the experiment, embryo mortality was recorded and the surviving embryos were fixed in $4 \%$ formaldehyde to determine whether there was any malformation. Head-tail lengths and malformations were evaluated under a light microscope and images were analyzed using Axiovision software version 4.8 (Carl Zeiss, Munich, Germany) to measure growth inhibition.

Whole-mount in situ hybridization. Xenopus embryos were prepared for whole-mount in situ hybridization using a previously described method (20), with minor modification. Whole-mount in situ hybridization was conducted at the tadpole stage (stage 34-36) using digoxigenin-labeled RNA probes and BM purple staining. The probes were prepared in the following manner: RT-PCR products of phosphotyrosine-binding protein (xPTB; a liver marker) and NKX2.5 (heart marker) 
Table I. Primers for RT-PCR.

\begin{tabular}{|c|c|c|c|c|c|}
\hline Gene name & $\begin{array}{l}\text { GeneBank } \\
\text { accession no. }\end{array}$ & RT-PCR primer sequences $\left(5^{\prime} \rightarrow 3^{\prime}\right)$ & Product size (bp) & $\begin{array}{c}\text { Annealing } \\
\text { temperature }\left({ }^{\circ} \mathrm{C}\right)\end{array}$ & Cycles \\
\hline xPTB & AY183756 & $\begin{array}{l}\text { F: ATGGATGCACTCAAGTCTGC } \\
\text { R: TGGTAGCTTCAGAAGTGTCAG }\end{array}$ & 933 & 58 & 28 \\
\hline NKX2.5 & NM_001172192 & $\begin{array}{l}\text { F: GTCAGCATCAACCCCTACAG } \\
\text { R: TGCCACCCAGTCCTTTAATC }\end{array}$ & 491 & 53 & 28 \\
\hline Cyl18 & AY188285 & $\begin{array}{l}\text { F: AGGCCGTAGCACCTGTGGGT } \\
\text { R: GGTCCCACCAGCTCGGATCA }\end{array}$ & 462 & 55 & 21 \\
\hline ODC & NM_001086698 & $\begin{array}{l}\text { F: GTGATGGGCTGGATCGTATC } \\
\text { R: TCCATTGAATGTCGAGGCTG }\end{array}$ & 386 & 55 & 21 \\
\hline
\end{tabular}

F, forward; R, reverse; RT-PCR, reverse transcriptase-polymerase chain reaction.

Table II. Teratogenic index for PFHxA and PFHpA.

\begin{tabular}{lrccc}
\hline Acid & $\begin{array}{c}\text { LC50 }(\mu \mathrm{M}) \\
\text { lethality }\end{array}$ & $\begin{array}{c}\text { EC50 }(\mu \mathrm{M}) \\
\text { malformation }\end{array}$ & MCIG $(\mu \mathrm{M})$ & TI=LC50/EC50 \\
\hline PFHxA & 1523.5 & 1046.5 & 412.3 & 1.43 \\
PFHpA & 942.4 & 754.9 & 297.4 & 1.25 \\
\hline
\end{tabular}

PFHxA, perfluorohexanoic acid; PFHpA, perfluoroheptanoic acid; TI, teratogenic index.

were sub-cloned into a T-easy vector (Promega, Fitchburg, WI, USA), linearized with ApaI and NcoI, respectively, and transcribed using a SP6 RNA polymerase kit (Invitrogen, Carlsbad, CA, USA).

$R T$-PCR. RNA was isolated from stage 34-46 embryos using TRIzol reagent, according to the manufacturer's instructions (Invitrogen), and quantified using an Optizen $320 \mathrm{UV}$ spectrometer (Mecacys, Deajeon, Korea). An aliquot of RNA (1 g) was reverse transcribed using a PrimeScript First-Strand cDNA Synthesis kit, according to the manufacturer's instructions (Takara, Shiga, Japan). Reactions containing template RNA without reverse transcriptase were prepared as the negative controls (no-RT) and carrier was used as the loading control. cDNA was amplified using specific PCR primer sets (Table I) and 2X Emerald Amp PCR Master mix (Takara). The PCR products were separated in a 1-1.2\% agarose gel and visualized on a transilluminator (Vilber Lourmat, Marne-la-Vallée, France) following ethidium bromide staining. The data were representative of at least three experiments. The Primer3 program was used to design primers and ODC was used as an internal control.

Extraction and analysis of the liver. Twenty-five embryos from each group were exposed to $\mathrm{PFHxA}$ and $\mathrm{PFHpA}$, respectively, for 10 days. The surviving tadpoles were fixed in $4 \%$ formaldehyde for $2 \mathrm{~h}$ at room temperature. Tadpoles (3-5) were randomly selected from each group and their livers removed under a light microscope (Zeiss Stemi 2000-c; Carl Zeiss). Images were captured and analyzed to monitor changes in the liver size.
Statistical analysis. Data for the three endpoints from each FETAX assay were analyzed using GraphPad Prism software (GraphPad Software, Inc., La Jolla, CA, USA). Images from the RT-PCR and whole-mount in situ hybridization assays were analyzed by ImageJ software (NIH). Statistical analyses were conducted using SPSS 13.0 software (version 18; SPSS, Inc., Chicago, IL, USA).

\section{Results}

Developmental toxicity and teratogenicity assessment of PFCs by FETAX assay. FETAX assays were performed to determine the potential teratogenicity and developmental toxicity of PFCs. FETAX assay results were expressed relatively in terms of the teratogenic index $(\mathrm{TI})=\mathrm{LC} 50 / \mathrm{EC} 50$, where LC50 was the concentration that at which $50 \%$ of the embryos were killed and EC50 was the concentration at which 50\% of the embryos were malformed. The minimum concentration that inhibits growth (MCIG) was also determined. Compounds were considered toxic when TI was $\geq 1.2$ (17).

We performed an initial FETAX assay to determine whether PFHxA and PFHpA had embryonic lethal or teratogenic effects. This result confirmed that PFHxA and PFHpA were potential teratogens and developmental toxicants. The LC50 value for PFHxA in Xenopus embryos was $1523.5 \mu \mathrm{M}$, while the EC50 value was $1046.5 \mu \mathrm{M}$, and the MCIG value was $412.3 \mu \mathrm{M}$, which produced a TI value of 1.43 , indicating that this compound was a potential teratogen and developmental toxicant. The LC50 value for PFHpA was $942.4 \mu \mathrm{M}$ in the Xenopus embryos, with an EC50 of $754.9 \mu \mathrm{M}$ and an MCIG value of $297.4 \mu \mathrm{M}$, producing a TI value of 1.25 . Thus, this compound was also considered a teratogen and developmental toxicant (Table II). PFHxA had a significantly higher TI value $(\mathrm{TI}=1.43)$ than PFHpA (TI=1.25). Furthermore, the LC50 and EC50 values were higher for PFHxA, which had a shorter fluorinated carbon chain than PFHpA.

Effects of PFHxA and PFHpA on Xenopus mortality and malformation rates. Our FETAX assay results confirmed that PFHxA and PFHpA are potential teratogens and developmental toxicants. We then investigated the effects of 
A<smiles>O=C(O)C(F)(F)C(F)(F)C(F)(F)C(F)(F)C(F)(F)F</smiles>

PFHXA<smiles>O=C(O)C(F)(F)C(F)(F)C(F)(F)C(F)(F)C(F)(F)C(F)(F)F</smiles>

PFHpA

B
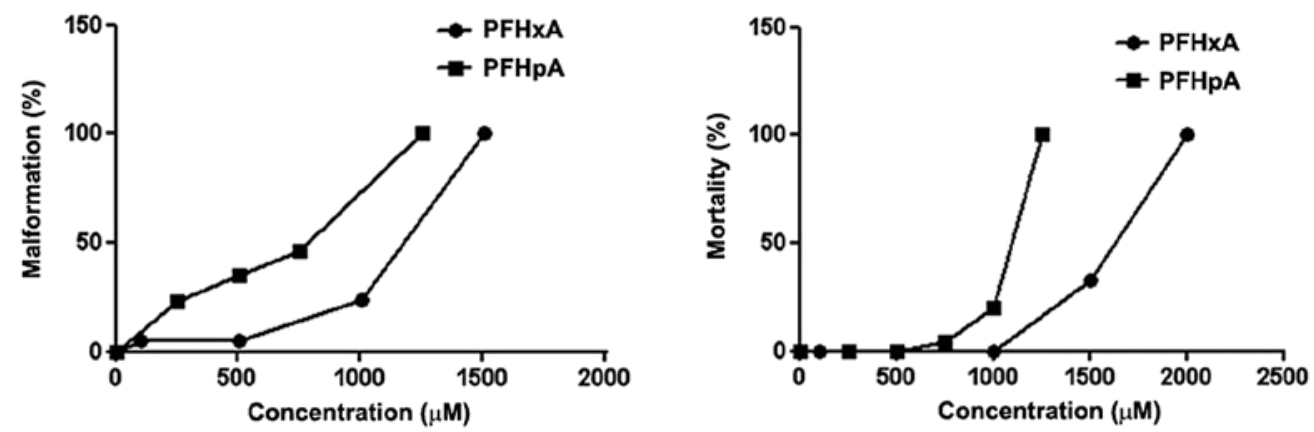

Figure 1. Lewis structures of PFCs. (A) Structures of PFHxA and PFHpA. (B) Effects of PFHxA and PFHpA on Xenopus mortality and malformation. Mortality and malformation rates were measured among embryos in stage 46 after exposure to PFHxA or PFHpA and the findings confirmed that the two compounds showed developmental toxicity and teratogenicity. PFCs, perfluoroalkyl compounds; PFHxA, perfluorohexanoic acid; PFHpA, perfluoroheptanoic acid.

A CTL

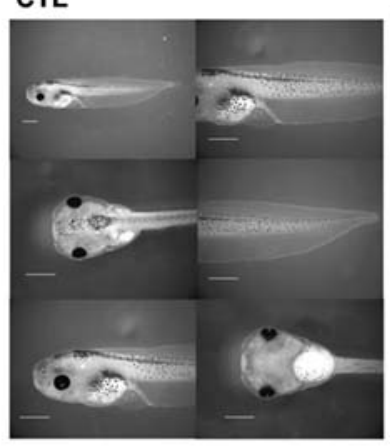

PFHXA

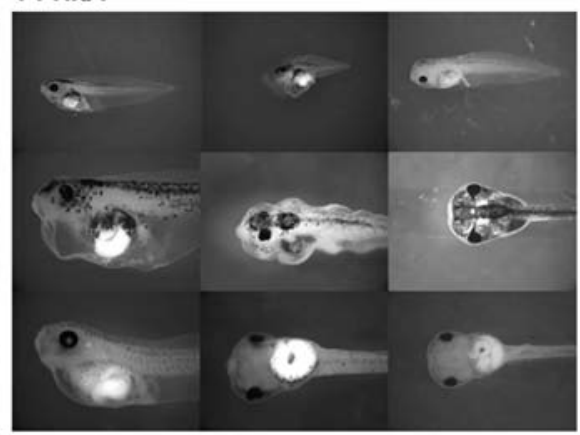

PFHpA

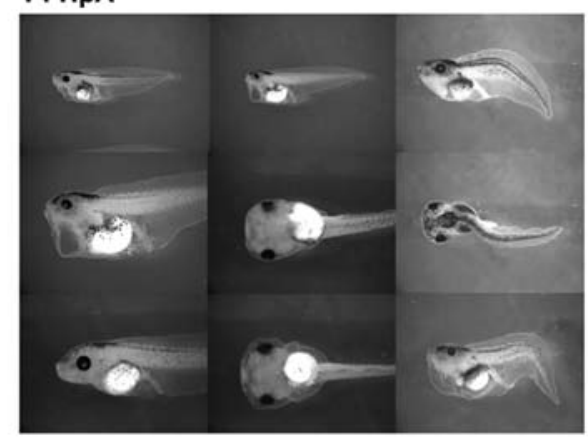

B

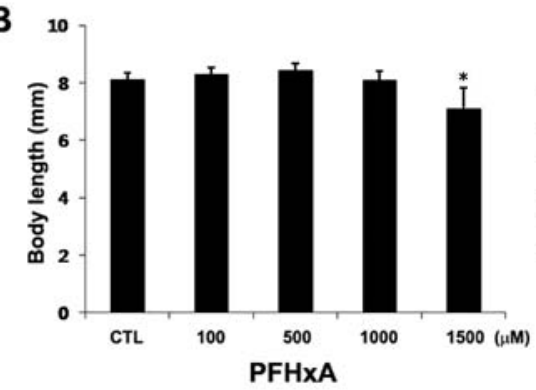

Figure 2. Abnormal phenotypes and shorter body lengths resulting from PFHxA and PFHpA exposure. Embryos developed various malformations including (A) stunted body, multiple edemas, gut mis-coiling, microcephaly, skeletal kinking and (B) shorter body length following exposure to PFHxA and PFHpA. Mean $\pm \mathrm{SD} ;{ }^{* *} \mathrm{P}<0.05,{ }^{*} \mathrm{P}<0.05$ compared with the control cells. PFHxA, perfluorohexanoic acid; PFHpA, perfluoroheptanoic acid; SD, standard deviation. 
A
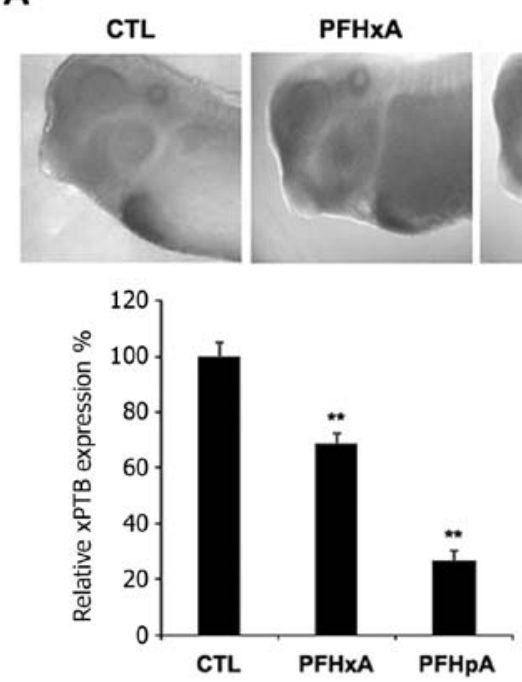

C

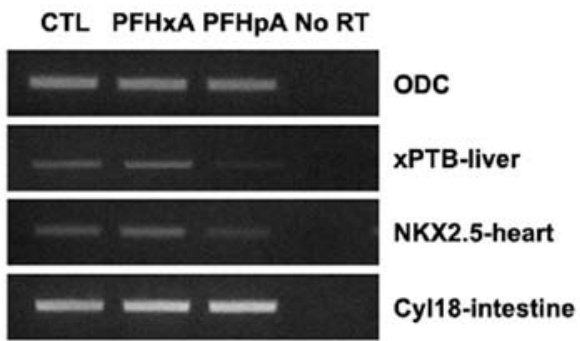

B
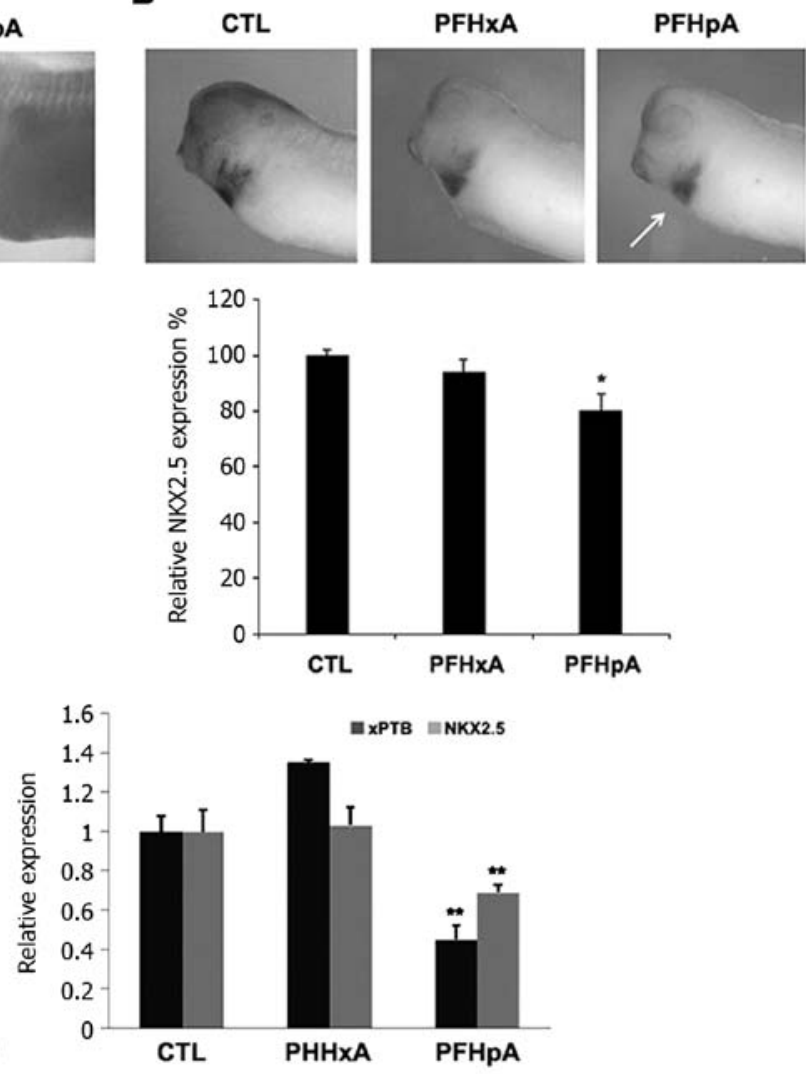

Figure 3. PFHpA severely affects liver and heart development. (A) PFHxA and PFHpA exposure altered the expression of tissue-specific mRNAs. Whole-mount in situ hybridization of stage 36 embryos exposed to $130 \mu \mathrm{M}$ PFHxA and PFHpA. xPTB expression levels were reduced in the liver and heart of exposed embryos (black arrow). The histogram shows the relative area and intensity of xPTB in the liver, expressed as a percentage of the control. Data are the mean of three independent experiments. (B) PFHxA and PFHpA exposure altered the expression of tissue-specific mRNAs. Whole-mount in situ hybridization of stage 36 embryos exposed to $130 \mu \mathrm{M}$ PFHxA and PFHpA. NKX2.5 expression levels were reduced in the heart of exposed embryos (white arrow). The histogram shows the relative area and intensity of NKX2.5 mRNA expression in the heart, expressed as a percentage of the control. Data are the mean of three independent experiments. (C) The levels of xPTB, NKX2.5, and Cyl18 mRNA expression were determined by RT-PCR. PCR was conducted without reverse transcriptase (No-RT) as a negative control and the carrier was used as the loading control. The histogram shows the relative expression levels of xPTB, NKX2.5, expressed as a percentage of the control ODC. Mean $\pm \mathrm{SD} ;{ }^{* *} \mathrm{P}<0.05,{ }^{*} \mathrm{P}<0.05$ compared with the control cells. PFHxA, perfluorohexanoic acid; PFHpA, perfluoroheptanoic acid; SD, standard deviation.

different concentrations of PFHxA and PFHpA on mortality and malformation in developing embryos. As shown in Fig. 1B, exposure to PFHxA or PFHpA greatly increased the mortality and general malformation rates in the developing embryos. These concentration-dependent effects on mortality and malformation were more severe as the number of carbons increased. PFHpA significantly affected mortality and malformation rates within a narrow concentration range (Fig. 1B).

To investigate the mechanism underlying this toxicity, we assessed the phenotypes of embryos exposed to PFHxA and PFHpA. PFHxA and PFHpA were not highly toxic until early stage 26, as no significant differences were observed in the external phenotypes. PFC exposure induced certain embryonic abnormalities that were observed at the end of the study, such as pericardial edema, dorsal fin blisters, a curved body axis, lack of eye pigment, and disturbed facial cells (Fig. 2A). PFHxA-treated embryos developed mild and general malformations including gut mis-coiling and improper eye shape, whereas PFHpA induced more severe and diverse malformations (Fig. 2A). In agreement with this, the whole body length was reduced by 14 and $24 \%$ in tadpoles treated with $1,500 \mu \mathrm{M}$ of PFHxA or 1,000 $\mu \mathrm{M}$ of PFHpA, respectively (Fig. 2B).
These results indicated that embryos exposed to PFHxA or PFHpA show a range of malformations and a reduced whole body length, which is a clear indicator of toxicity while PFHpA has slightly more severe defects compared to PFHxA.

PFHpA modulates liver and heart development during embryogenesis. We examined stage 36 tadpoles that had been continuously exposed to $130 \mu \mathrm{M}$ PFHxA or PFHpA using wholemount in situ hybridization. The liver is a major metabolizing organ that detoxifies many compounds and is therefore vulnerable to the toxic effects of PFCs. We also measured a liver-specific marker, $\mathrm{xPTB}$, which is expressed in the diverticulum region of the embryonic liver and plays an important role in low-density lipoprotein (LDL) receptor endocytosis in the liver (21). Livers of embryos treated with PFHxA developed abnormally and were unusually large (Fig. 3A). We also observed enlarged livers in tadpoles exposed to PFHxA although these effects were more pronounced in the presence of PFHpA (Fig. 2A). These results indicated that PFHxA and PFHpA induced liver defects and should be regarded as developmental toxicants and teratogens.

The heart-specific marker, NKX2.5, was used to examine the cardiovascular effects of PFC exposure. The amphibian 


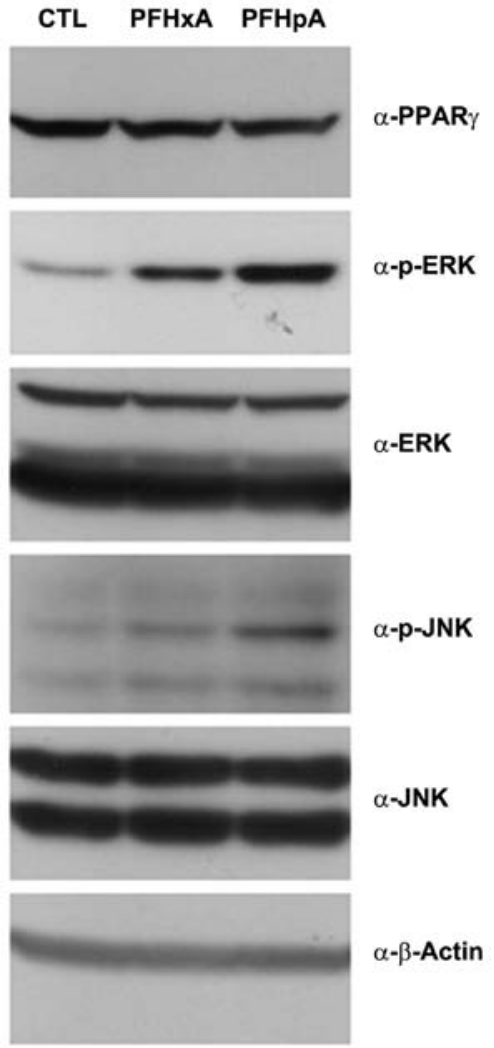

Figure 4. PFCs may induce liver toxicity by modifying ERK and JNK phosphorylation. Xenopus embryos were exposed to $130 \mu \mathrm{M}$ PFHxA or PFHpA Total protein was isolated and analyzed by western blotting with antibodies raised against PPAR $\gamma$, p-ERK, ERK, p-JNK, JNK, or $\beta$-actin. PFHxA and PFHpA treatment activated PPAR $\gamma$ and ERK/JNK phosphorylation. PFCs, perfluoroalkyl compounds; ERK, extracellular signal-regulated kinase; JNK, c-Jun N-terminal kinase; PFHxA, perfluorohexanoic acid; PFHpA, perfluoroheptanoic acid; PPAR $\gamma$, peroxisome proliferator-activated receptor- $\gamma$.

heart is composed of one ventricle, surrounded by atria on both sides, separated by a septum (22). Embryos treated with PFHxA and PFHpA showed enlarged atria and a loss of the atrial septum (Fig. 3B). Hearts from embryos exposed to either PFHxA or PFHpA were smaller and contained no trabeculae. Additionally, the pericardiac cavity was expanded and the atrial and ventricular walls were thinner than those observed in the control embryo hearts, which contained prominent trabeculae and the atria and ventricle were separated (Fig. 3B). This effect was more prominent in embryos exposed to PFHpA, which reduced trabeculation of the ventricle as well as the thickness of the atrial and ventricular walls (Fig. 3B). The results showed that PFHxA and PFHpA treatment caused abnormal heart development.

PFC exposure alters tissue-specific mRNA expression in vivo. To investigate the effects of PFC exposure on the liver and heart further, the mRNA expression levels of three liver, heart, and intestine markers were determined by RT-PCR analysis. Previously, PFCs were shown to be tissue-specific toxicants $(14,23,24)$. For this study, we exposed stage 36 embryos to $130 \mu \mathrm{M}$ PFHxA or PFHpA, a concentration that induced the development of various malformations, rather than embryonic lethality. As shown in Fig. 2C, mRNA expression of xPTB was considerably reduced in PFHpA-treated embryos, as compared to the control or PFHxA-treated embryos (Fig. 3A and C). Previous findings have also shown reduced $\mathrm{XPTB}$ expression following exposure to various PFCs $(16,25,26)$. Furthermore, the heart-specific marker, NKX2.5, was used to examine the effects of PFCs on the heart. As shown in Fig. 2B and C, mRNA expression of the heart-specific marker, NKX2.5, was reduced in the PFHpA-treated embryos (Fig. 3B and C). NKX2.5 is critical for vasculogenesis and angiogenesis (27). We also assessed the mRNA levels of an intestinal marker, Cyl18, which is mainly expressed in the presumptive small intestine of embryos at the tailbud stage. As shown in Fig. 2C, we observed no significant effects of either PFHxA or PFHpA exposure on Cy118 expression. These results suggested that PFHpA negatively affected liver and heart development (Fig. 3C).

PFCs may induce liver toxicity by modifying ERK and $J N K$ phosphorylation. The roles of peroxisome proliferator-activated receptor- $\gamma$ (PPAR $\gamma)$, ERK, and JNK pathways in mediating the developmental toxicity associated with exposure to PFCs remain to be elucidated. To investigate the effects of PFCs on PPAR $\gamma$, ERK, and JNK pathways, stage 36 embryos were exposed to $130 \mu \mathrm{M}$ PFHxA or PFHpA and then analyzed by western blotting with anti-PPAR $\gamma$, phosphorylated-ERK, ERK, p-JNK, JNK, and $\beta$-actin antibodies. PFHpA strongly induced ERK and JNK phosphorylation while PPAR $\gamma$ expression remained the same, which in turn activated signaling that caused liver toxicity, known as hepatocarcinogenesis (Fig. 4). These results clearly demonstrated that the observed developmental defects in the liver were associated with the PFHpA-mediated activation of ERK/JNK pathways.
CTL

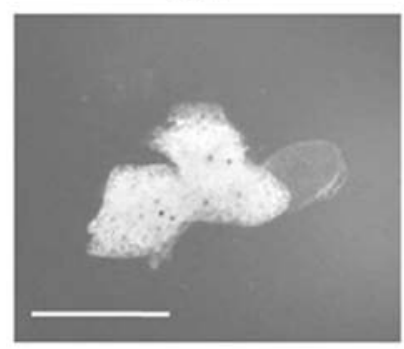

PFHxA

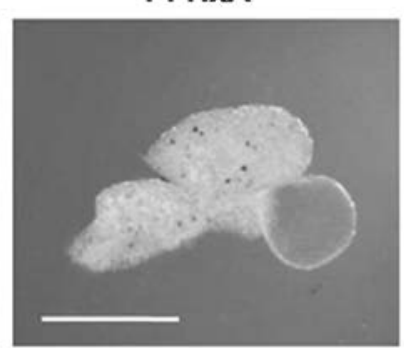

PFHpA

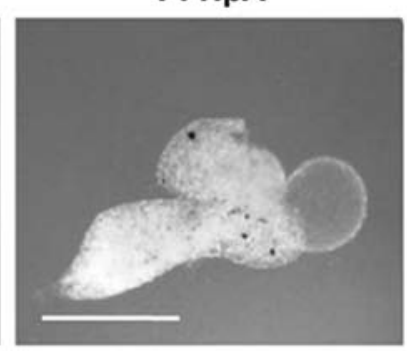

Figure 5. PFCs treatment induced hepatomegaly. Livers of tadpoles exposed to PFHxA or PFHpA. Livers of embryos treated with PFHxA and PFHpA were swollen, with severe morphological changes that were not observed in the control embryos. PFCs, perfluoroalkyl compounds; PFHxA, perfluorohexanoic acid; PFHpA, perfluoroheptanoic acid. 
PFC treatment induces hepatomegaly. Hepatomegaly is often associated with chronic inflammatory liver disease. The liver is the major metabolizing organ and it is targeted following PFC exposure (28). Previous findings have shown that exposure to PFCs caused various chronic abnormalities, including hepatocellular hypertrophy and hepatomegaly $(29,30)$. To investigate the effects of PFHxA and PFHpA on the liver, livers from 10-day old tadpoles that had been continuously exposed to $130 \mu \mathrm{M}$ PFHxA or PFHpA exhibited hepatomegaly (Fig. 5). These results suggested that high levels of exposure to PFHpA or PFHxA may induce hepatic disorders.

\section{Discussion}

PFCs have emerged as persistent organic pollutants in the ecosystem and human exposure to these may increase the risk of developmental abnormalities (31). Several studies have examined the toxicity of different PFC in many aquatic and other animals $(24,32,33)$. These studies have confirmed that exposure during particular developmental stages or under certain nutritional conditions produced adverse effects on neonates $(34,35)$. Amphibians are more sensitive to environmental exposures during the fetal stage. The results of that study showed that the PFCs tested induced malformations in Xenopus embryos, including pericardial edema and hepatic defects. The industrial production of PFCs and their derivatives has been banned because of their toxicity and persistence in the environment. Given these conditions, developmental toxicity studies of PFCs remain in their infancy and further large-scale investigations are required.

Xenopus laevis is an amphibian species considered to be a suitable model for studying developmental defects induced by toxicants. In the present study, we conducted a FETAX assay using Xenopus laevis to examine the developmental toxicities of PFHxA and PFHpA. The FETAX assay provides a classical approach to detecting teratogenic effects of chemicals on fertilized Xenopus embryos in the middle of the blastula stage (17). When Xenopus embryos were exposed to C6-7 PFCs, various malformations and toxicity were observed, such as abnormal development, bent and uneven body axis deformation, multiple edemas, growth inhibition, and intestinal miscoiling (Fig. 2A). The PFCs tested produced different TI values, showing that the intensity of their effects on development were different, although they may operate via identical mechanisms.

Our results have demonstrated that PFHxA and PFHpA acted as developmental toxicants and teratogens, thereby producing lethal effects in Xenopus embryos. The difference between their effects may reflect the variation in the number of fluorinated carbons present in these compounds. In addition, PFHxA and PFHpA considerably modulated the expression levels of various tissue-specific genes that were relevant to the observed external and internal phenotypes. In particular, XPTB was used as a liver-specific marker and the protein encoded by this gene complexes with the LDL receptor to mediate hepatic LDL removal by endocytosis $(35,36)$. Consistent with other reports, the xPTB mRNA levels were considerably modulated following exposure to either PFHxA or PFHpA (16). This specific reduction in the XPTB mRNA level may cause increased LDL accumulation in the liver, which can in turn lead to chronic cardiac disease, such as atherosclerosis and myocardial infarction (37). Consistent with this, individuals with a high concentration of PFCs in their blood are more susceptible to LDL-associated adverse effects (5).

The amphibian heart develops from the mesoderm and is considered an essential organ for embryonic survival. During embryogenesis, NKX2.5 acts as a key transcription factor, regulating the transcription of downstream targets that are essential for heart formation $(38,39)$. Studies have shown that NKX2.5 has an important role in the formation of the septum, which separates the ventricle from the atrium $(15,38,39)$. Severe heart abnormalities such as atrial septal defects have been reported in association with the NKX2.5 mutation and downregulation. PFHxA and $\mathrm{PFHpA}$ treatments reduced the expression of NKX2.5 mRNA, which is critical for vasculogenesis and angiogenesis (32). Thus, PFCs may cause improper septum formation and impair cardiac function by downregulating the expression of NKX2.5. By contrast, no major change was observed in the intestine-specific marker, Cy118, following treatment with PFHxA and PFHpA.

Compelling evidence for PFC toxicity has emerged from pre-clinical and clinical studies $(12,18)$. Activation of JNK and ERK pathway causes toxic effects that include hepatocarcinogenesis. Livers from PFHxA- and PFHpA-treated embryos exhibited hepatomegaly. This developmental and teratogenic effect was more pronounced in the presence of PFHpA, which may be due to its longer fluorinated carbon chain.

In conclusion, our results confirm that PFHxA and PFHpA are developmental toxicants and teratogens in Xenopus. In addition, PFHpA induced more severe pericardial edema, blisters on the dorsal fin, a curved body axis, lack of eye pigmentation, and disturbed facial cells. Furthermore, PFHpA produced more severe effects on liver and heart development through the induction of ERK and JNK phosphorylation, and exposure to elevated levels of PFHpA may therefore cause developmental toxicity and teratogenicity, inducing hepatic and cardiac disorders.

\section{Acknowledgements}

The present study was supported by the Basic Science Research Program through the National Research Foundation of Korea (NRF), funded by the Ministry of Education, Science and Technology (no. NRF-2012R1A1A2042334), and by a grant from the National Fisheries Research and Development Institute (NFRDI; contribution no. RP-2015-AQ-072), Republic of Korea.

\section{References}

1. Andersen ME, Butenhoff JL, Chang SC, Farrar DG, Kennedy GL Jr, Lau C, Olsen GW, Seed J and Wallace KB: Perfluoroalkyl acids and related chemistries - toxicokinetics and modes of action. Toxicol Sci 102: 3-14, 2008.

2. Houde M, Martin JW, Letcher RJ, Solomon KR and Muir DC: Biological monitoring of polyfluoroalkyl substances: A review. Environ Sci Technol 40: 3463-3473, 2006.

3. Calafat AM, Wong LY, Kuklenyik Z, Reidy JA and Needham LL: Polyfluoroalkyl chemicals in the U.S. population: Data from the National Health and Nutrition Examination Survey (NHANES) 2003-2004 and comparisons with NHANES 1999-2000. Environ Health Perspect 115: 1596-1602, 2007.

4. Lindstrom AB, Strynar MJ and Libelo EL: Polyfluorinated compounds: Past, present, and future. Environ Sci Technol 45: 7954-7961, 2011. 
5. Nelson JW, Hatch EE and Webster TF: Exposure to polyfluoroalkyl chemicals and cholesterol, body weight, and insulin resistance in the general U.S. population. Environ Health Perspect 118: 197-202, 2010.

6. Yamashita N, Kannan K, Taniyasu S, Horii Y, Petrick G and Gamo T: A global survey of perfluorinated acids in oceans. Mar Pollut Bull 51: 658-668, 2005.

7. McLachlan MS, Holmstrom KE, Reth M and Berger U: Riverine discharge of perfluorinated carboxylates from the European continent. Environ Sci Technol 41: 7260-7265, 2007.

8. Jin YH, Liu W, Sato I, Nakayama SF, Sasaki K, Saito N and Tsuda S: PFOS and PFOA in environmental and tap water in China. Chemosphere 77: 605-611, 2009.

9. Nakayama S, Strynar MJ, Helfant L, Egeghy P, Ye X and Lindstrom AB: Perfluorinated compounds in the Cape Fear Drainage Basin in North Carolina. Environ Sci Technol 41: 5271-5276, 2007.

10. Domingo JL: Health risks of dietary exposure to perfluorinated compounds. Environ Int 40: 187-195, 2012.

11. Moody CA, Martin JW, Kwan WC, Muir DC and Mabury SA: Monitoring perfluorinated surfactants in biota and surface water samples following an accidental release of fire-fighting foam into Etobicoke Creek. Environ Sci Technol 36: 545-551, 2002.

12. Olsen GW, Butenhoff JL and Zobel LR: Perfluoroalkyl chemicals and human fetal development: An epidemiologic review with clinical and toxicological perspectives. Reprod Toxicol 27: 212-230, 2009

13. Dreyer A, Weinberg I, Temme C and Ebinghaus R: Polyfluorinated compounds in the atmosphere of the Atlantic and Southern Oceans: Evidence for a global distribution. Environ Sci Technol 43: 6507-6514, 2009.

14. Lau C, Anitole K, Hodes C, Lai D, Pfahles-Hutchens A and Seed J: Perfluoroalkyl acids: A review of monitoring and toxicological findings. Toxicol Sci 99: 366-394, 2007.

15. Kato K, Wong LY, Jia LT, Kuklenyik Z and Calafat AM: Trends in exposure to polyfluoroalkyl chemicals in the U.S. Population: 1999-2008. Environ Sci Technol 45: 8037-8045, 2011.

16. Kim M, Son J, Park MS, Ji Y, Chae S, Jun C, Bae JS, Kwon TK, Choo YS, Yoon $\mathrm{H}$, et al: In vivo evaluation and comparison of developmental toxicity and teratogenicity of perfluoroalkyl compounds using Xenopus embryos. Chemosphere 93 $1153-1160,2013$.

17. Mouche I, Malesic L and Gillardeaux O: FETAX assay for evaluation of developmental toxicity. Methods Mol Biol 691: 257-269, 2011.

18. Sharma B and Patiño R: Exposure of Xenopus laevis tadpoles to cadmium reveals concentration-dependent bimodal effects on growth and monotonic effects on development and thyroid gland activity. Toxicol Sci 105: 51-58, 2008

19. Bacchetta R, Santo N, Fascio U, Moschini E, Freddi S, Chirico G Camatini $\mathrm{M}$ and Mantecca P: Nano-sized $\mathrm{CuO}, \mathrm{TiO}_{2}$ and $\mathrm{ZnO}$ affect Xenopus laevis development. Nanotoxicology 6: 381-398, 2012.

20. Vize PD, McCoy KE and Zhou X: Multichannel wholemount fluorescent and fluorescent/chromogenic in situ hybridization in Xenopus embryos. Nat Protoc 4: 975-983, 2009.

21. Zhou Y, Zhang J and King ML: Xenopus autosomal recessive hypercholesterolemia protein couples lipoprotein receptors with the AP-2 complex in oocytes and embryos and is required for vitellogenesis. J Biol Chem 278: 44584-44592, 2003.

22. Jahr M and Männer J: Development of the venous pole of the heart in the frog Xenopus laevis: A morphological study with special focus on the development of the venoatrial connections. Dev Dyn 240: 1518-1527, 2011
23. Ruch RJ: Intercellular communication, homeostasis, and toxicology. Toxicol Sci 68: 265-266, 2002.

24. Cheng Y, Cui Y, Chen HM and Xie WP: Thyroid disruption effects of environmental level perfluorooctane sulfonates (PFOS) in Xenopus laevis. Ecotoxicology 20: 2069-2078, 2011.

25. Ny A, Koch M, Schneider M, Neven E, Tong RT, Maity S, Fischer C, Plaisance S, Lambrechts D, Héligon C, et al: A genetic Xenopus laevis tadpole model to study lymphangiogenesis. Nat Med 11: 998-1004, 2005.

26. Kälin RE, Bänziger-Tobler NE, Detmar M and Brändli AW: An in vivo chemical library screen in Xenopus tadpoles reveals novel pathways involved in angiogenesis and lymphangiogenesis. Blood 114: 1110-1122, 2009.

27. Raffin M, Leong LM, Rones MS, Sparrow D, Mohun T and Mercola M: Subdivision of the cardiac Nkx2.5 expression domain into myogenic and nonmyogenic compartments. Dev Biol 218: 326-340, 2000.

28. Son HY, Lee S, Tak EN, Cho HS, Shin HI, Kim SH and Yang JH: Perfluorooctanoic acid alters $\mathrm{T}$ lymphocyte phenotypes and cytokine expression in mice. Environ Toxicol 24: 580-588, 2009.

29. Van den Heuvel LP, Van den Born J, Jalanko H, Schröder CH Veerkamp JH, Assmann KJ, Berden JH, Holmberg C, Rapola J and Monnens LA: The glycosaminoglycan content of renal basement membranes in the congenital nephrotic syndrome of the Finnish type. Pediatr Nephrol 6: 10-15, 1992.

30. Yeung LW, Yamashita N, Taniyasu S, Lam PK, Sinha RK, Borole DV and Kannan K: A survey of perfluorinated compounds in surface water and biota including dolphins from the Ganges River and in other waterbodies in India. Chemosphere 76: 55-62, 2009.

31. Shi X, Du Y, Lam PK, Wu RS and Zhou B: Developmental toxicity and alteration of gene expression in zebrafish embryos exposed to PFOS. Toxicol Appl Pharmacol 230: 23-32, 2008.

32. Abbott BD, Wolf CJ, Schmid JE, Das KP, Zehr RD, Helfant L, Nakayama S, Lindstrom AB, Strynar MJ and Lau C: Perfluorooctanoic acid induced developmental toxicity in the mouse is dependent on expression of peroxisome proliferator activated receptor-alpha. Toxicol Sci 98: 571-581, 2007.

33. O'Brien JM, Crump D, Mundy LJ, Chu S, McLaren KK, Vongphachan V, Letcher RJ and Kennedy SW: Pipping success and liver mRNA expression in chicken embryos exposed in ovo to $\mathrm{C} 8$ and $\mathrm{C} 11$ perfluorinated carboxylic acids and $\mathrm{C} 10$ perfluorinated sulfonate. Toxicol Lett 190: 134-139, 2009.

34. Verreault J, Houde M, Gabrielsen GW, Berger U, Haukås M, Letcher RJ and Muir DC: Perfluorinated alkyl substances in plasma, liver, brain, and eggs of glaucous gulls (Larus hyperboreus) from the Norwegian arctic. Environ Sci Technol 39: 7439-7445, 2005.

35. Conder JM, Hoke RA, De Wolf W, Russell MH and Buck RC: Are PFCAs bioaccumulative? A critical review and comparison with regulatory criteria and persistent lipophilic compounds. Environ Sci Technol 42: 995-1003, 2008.

36. Garuti R, Jones C, Li WP, Michaely P, Herz J, Gerard RD, Cohen JC and Hobbs HH: The modular adaptor protein autosomal recessive hypercholesterolemia (ARH) promotes low density lipoprotein receptor clustering into clathrin-coated pits. J Biol Chem 280: 40996-41004, 2005.

37. Goldstein JL and Brown MS: Molecular medicine. The cholesterol quartet. Science 292: 1310-1312, 2001.

38. Huang Q, Fang C, Wu X, Fan J and Dong S: Perfluorooctane sulfonate impairs the cardiac development of a marine medaka (Oryzias melastigma). Aquat Toxicol 105: 71-77, 2011.

39. Jiang Q, Lust RM, Strynar MJ, Dagnino S and DeWitt JC: Perflurooctanoic acid induces developmental cardiotoxicity in chicken embryos and hatchlings. Toxicology 293: 97-106, 2012. 